\title{
Live Demonstration: A Closed-loop Cortical Brain Implant for Optogenetic Curing Epilepsy
}

Jun wen Luo, Dimitrios Firfilionis, Reza Ramezani,

Fahimeh Dehkhoda, Ahmed Soltan and Patrick Degenaar

School of Electrical and Electronic Engineering

Newcastle University, UK

\section{DEMONSTRATION SETUP}

A closed-loop optogenetic system for curing epilepsy is presented in this work. As it shown at figure 1, the system consists of a cortical brain implant with LEDs and recording electrodes, a customer designed CMOS chip[1][2][3] and a controller. The brain activities are recorded by the implant with recording electronics in a CMOS chip, the signals are processed by the controller, and the results are send back to the CMOS chip for delivering LED stimulation commands.

\section{VISITOR EXPERIENCE}

There are three major technologies that visitor may have strong interests: 1) closed-loop processing: by real-time monitoring recording and stimulations in a fully functional prototype system, the visitors can obtain the conceptual idea that how presented technology can be applied on human beings to cure epilepsy. 2) Algorithms implementation: three different types of algorithm (threshold based, manual control and phaseshift) in the controller can be selected directly by visitor via Matlab GUI interface (figure 2). The results will be displayed in the GUI LED commands window immediately. This will enhance visitor interactive experiences. 3) self-diagnosis: the implantable LED status can be observed in the GUI as well.

\author{
Yan Liu and Timothy Constandinou \\ Department of Electrical and Electronic Engineering \\ Imperial College London, \\ London, UK
}

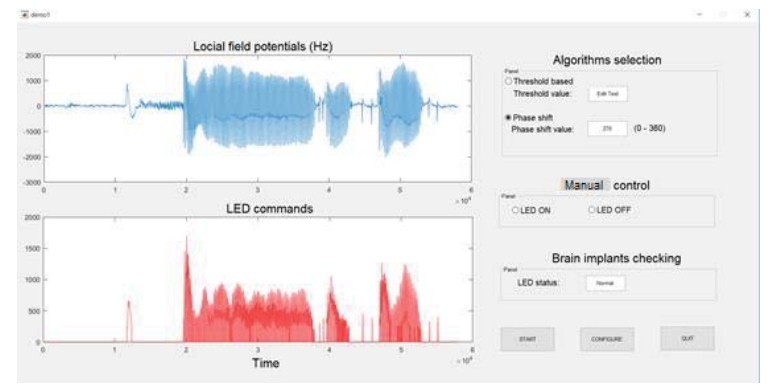

Figure 2: The system user interface.

\section{EARLIER PUBLICATION}

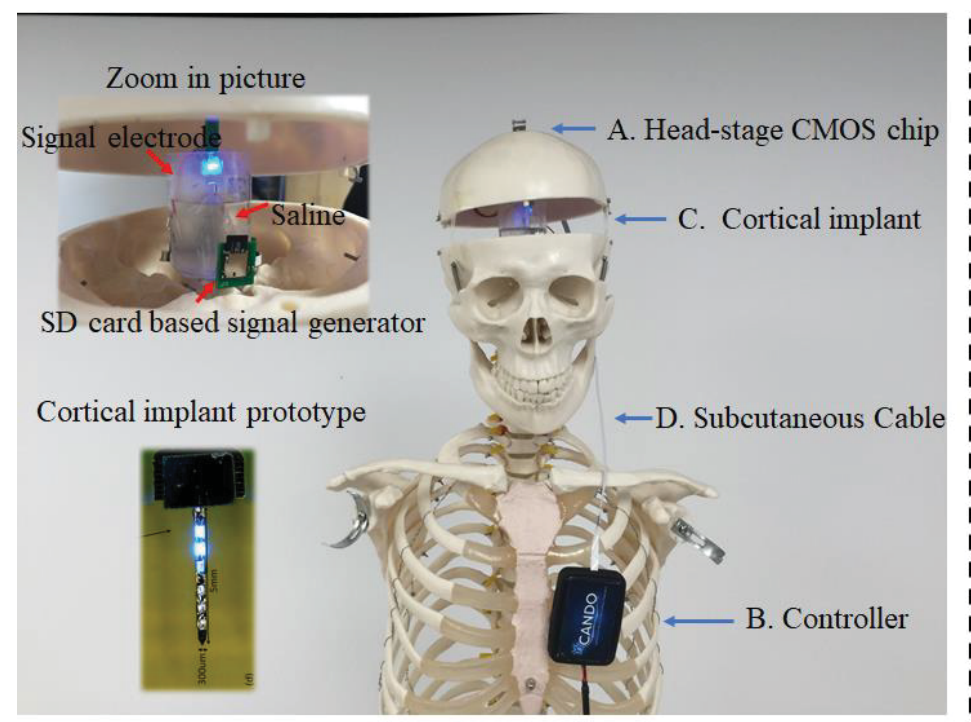

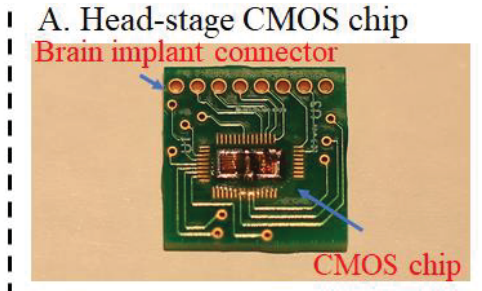

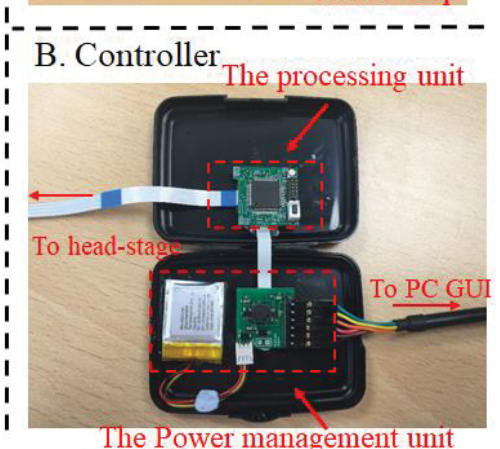

Figure 1.: The system overview. A is the custom designed CMOS chip, B is a controller that contains a processor (ARM cortexM4), a power management system and a battery; $\mathrm{C}$ is a cortical brain implant with one LED and one recording electrode and D is a 10-pin position subcutaneous cable. The zoom in picture displays SD card based system generates seizure-like events signals. 